\title{
Association between metal hypersensitivity and implant failure in patients who underwent titanium cranioplasty
}

\author{
${ }^{*}$ Yirui Sun, MD, PhD, ${ }^{1}$ Yue Hu, MD, ${ }^{2}$ Qiang Yuan, MD, PhD, Jian Yu, MD, PhD, ${ }^{1}$ Xing Wu, MD, PhD, ${ }^{1}$ \\ Zhuoying $\mathrm{Du}, \mathrm{MD}, \mathrm{PhD},{ }^{1}$ Xuehai $\mathrm{Wu}, \mathrm{MD}, \mathrm{PhD},{ }^{1}$ and $\mathrm{Jin} \mathrm{Hu}, \mathrm{MD}, \mathrm{PhD}^{1}$
}

Departments of ${ }^{1}$ Neurosurgery and ${ }^{2}$ Dermatology, Huashan Hospital, Fudan University, Shanghai, People's Republic of China

OBJECTIVE Digitally designed titanium plates are commonly used for the reconstruction of craniofacial defects, although implant exposure (referred to as failure) is one of the major complications. Metal hypersensitivities have been suggested as possible causes of implant failure of orthopedic, intravascular, gynecological, and dental devices, yet there has been no consensus on the requirement for allergy screening before cranioplasty.

METHODS In this study, the authors prospectively investigated the prevalence of metal hypersensitivity in patients for whom cranioplasty is planned and assess its relationship with titanium implant failure (exposure).

RESULTS Based on records from 207 included patients, 39.61\% of patients showed hypersensitivity to at least one kind of metal. Approximately one-quarter (25.12\%) of patients had multiple metal allergies. Co, Cd, and Zn were the 3 most frequently identified metal hypersensitivities. No allergy to titanium was detected in this study. The overall incidence of cranioplasty implant failure was 5.31\% (11 of 207). Patients showing hypersensitivities to more than 3 kinds of metal had higher risks of titanium plate exposure.

CONCLUSIONS Based on their findings, the authors suggest that routine allergy screening be performed before titanium plate cranioplasty. For patients with hypersensitivities to more than 3 metals, alternative materials, such as polyetheretherketone, should be considered for cranioplasty.

https://thejns.org/doi/abs/10.3171/2018.1.JNS171804

KEYWORDS cranioplasty; metal hypersensitivity; implant failure; patch test; surgical technique

$\mathrm{C}$ RANIOPLASTY is performed for skull defects resulting from neurotrauma, decompressive craniectomy, and other etiologies. The purposes of cranioplasty usually include restoring the contour of the skull, preserving the rigid and protective integrity of the calvaria, and minimizing morbidity and potential complications. Recently, studies have also shown that cranioplasty may also accelerate and improve neurological recovery. ${ }^{1,5,10}$ Cranioplasty with an autogenous bone flap carries the risk of resorption and requires additional storage strategies after decompressive craniectomies, such as freezer cryopreservation or subcutaneous abdominal implantation. .,9,12 $^{2}$ Computer-designed polyetheretherketone (PEEK) reconstruction has recently been proven as an effective and safe cranioplasty technique, but the price of each PEEK unit is expensive, particularly for uninsured patients in developing countries..$^{15,19}$ Therefore, metal implants, such as titanium plates, are commonly used for cranioplasty in many countries, including mainland China. Previous epidemiological investigations have suggested that over $95 \%$ of patients receive CT-modeled titanium implants during cranioplasty, which has been proven to be a precise, time-saving, and affordable technique. ${ }^{2,16,23}$

One significant complication of titanium cranioplasty is implant failure, which often leads to exposure of the metal plates or screws, requiring additional operations for removal. The incidence of cranioplasty failure has been reported to be between $4 \%$ and $25 \%$. $^{13,19,21,22}$ Infections were often presumed as a major cause of cranioplasty failure, but recent studies have suggested that such complications

ABBREVIATIONS PEEK = polyetheretherketone.

SUBMITTED July 26, 2017. ACCEPTED January 19, 2018.

INCLUDE WHEN CITING Published online July 6, 2018; DOI: 10.3171/2018.1.JNS171804.

${ }^{*}$ Y.S., Y.H., and Q.Y. contributed equally to this work. 
may also be caused or accelerated by host immune sensitivity to metal substances within the implant. Metal hypersensitivities may lead to cutaneous eczematous eruptions, chronic inflammation, pain, and a range of other adverse reactions, such as device breakage or exposure, which have been noted in orthopedic, endovascular, obstetric, and dental surgeries..$^{18}$ Although consensus has not been achieved, it is theorized that skin patch testing may be an accurate way to identify this immune sensitivity and that this test can be used to guide implant material selection and the potential need for implant removal. ${ }^{4,17}$ However, the incidence of metal hypersensitivities in patients requiring cranioplasty has not been investigated. Metal mesh/plates for cranioplasty are usually made of titanium alloy (91\%-99\% titanium). Hypersensitivities to titanium or other metals within the alloy may preclude their use as implantable material, either as a cranioplasty plate or as screw fixation for autologous bone or an alloplastic plate. The aims of this study were to investigate the incidence of metal hypersensitivity in patients scheduled for cranioplasty and to assess the relationship between metal allergy and titanium alloy implant failure (exposure).

\section{Methods \\ Patient Population}

Between September 2012 and January 2017, a prospective cohort study was conducted at 2 independent medical institutions: Shanghai Huashan Hospital and Shanghai Minhang Hospital, both of which are university clinical institutions. Approval was obtained from the institutional review boards. Informed consent was acquired from all patients and/or their closest direct relative. The study approaches were approved by the ethics committee and the Chinese Clinical Trial Registry. Patients $\geq 14$ years with skull defects who were scheduled for titanium alloy cranioplasty were recruited. Patients with a history of metal dermatitis were excluded and were advised to receive PEEK cranioplasty. Patients who underwent decompressive craniectomy due to malignant intracranial tumors, such as glioma, were not included, since tumor recurrence, chemotherapy, and potential secondary operations could affect the follow-up observations. Patients with the following conditions were also excluded from this study: pregnancy, rheumatoid arthritis currently under corticosteroid or other immunosuppressive therapy, and other contraindications to either cranioplasty or patch testing.

\section{Patch Testing}

All included patients underwent skin patch tests at 3 appointments, which were carried out by 2 independent dermatologists. Application of the patch tests takes about 15 minutes at the first appointment. Very small quantities of allergens in individual square plastic are applied to the upper back and are kept in place with hypoallergenic adhesive tape. The patches stay in place for at least 48 hours, during which period patients were advised to avoid vigorous exercise, stretching, and showering. At the second appointment, usually 48 hours later, the patches are removed. The patient's back is marked with an indelible black felt-tip pen to identify the test sites. These marks must be visible at the third appointment, usually 72-96 hours after initial application. The dermatologists recorded findings at the second and third appointments. The results for each test site were recorded as follows: negative (-); weak erythema only/uncertain $( \pm)$; erythema and edema $(+)$; erythema and edema with papules and vesicles confined within the chamber (++); and erythema with vesicles, papules, or bullae extending beyond the chamber (+++). Metals that are most frequently seen in metallic alloy implants were tested as allergens, including $0.5 \%$ potassium dichromate, $5 \%$ nickel nitrate, $1 \%$ cobalt chloride, $10 \%$ titanium chloride, $2 \%$ palladium chloride, $5 \%$ aluminum nitrate, $2 \%$ manganese chloride, $2 \%$ iron trichloride, $1 \%$ ammonium molybdate tetrahydrate, $1 \%$ cadmium chloride, $2 \%$ mercuric chloride, $2 \%$ silver nitrate, and $2 \%$ zinc sulfate. All allergens were dispersed in Vaseline, with the exception of ammonium molybdate tetrahydrate, which was dispersed in water. Vaseline was applied as a negative control.

\section{Cranioplasty and Implant Failure}

Cranioplasty was performed at least 8 weeks after initial decompressive craniectomy, with the exception of the 2 cases of osteofibrous dysplasia in which cranioplasty was performed immediately after the craniectomy. Patients were free to choose a titanium plate or PEEK for cranioplasty. The patch test results and the possibility of implant failure were well explained to all patients. Informed consent was acquired from all patients and/or their closest direct relative. To achieve precision-fitting prostheses, cranial defects were modeled from CT scanning data using stacked slices, computer-driven milling, and selective laser sintering. Cranioplasty with autologous bone was not performed at our institution. Follow-up visits were conducted for 12 months after cranioplasty.

Cranioplasty failure was defined as removal of a patient's implant due to any of the following reasons: 1) superficial infection, involving skin and subcutaneous tissues of the incision site; 2) deep infection, involving deep soft tissues, meninges, or brain; and 3) exposed implant, defined as exposure of implant due to impaired wound healing or erosion of the skin.

\section{Statistical Analysis}

Statistical analysis was carried out using Stata 13 (StataCorp LP) and Excel 2013 (Microsoft Corp.) software. Quantitative data are expressed as the mean \pm standard deviation (SD). The Shapiro-Wilk W test and F test were performed for normality and homogeneity of variance. The independent-samples t-test, Satterthwaite t-test, Wilcoxon rank-sum test, and 1-way ANOVA were used for the quantitative data of independent groups. The chi-square test was used for qualitative data. Fisher's exact test was used in the analysis of contingency tables where sample sizes are small $(n<40)$. Statistical inference was conducted at a significance level of 0.05 .

\section{Results}

\section{Patient Demographics}

During the period between September 2012 and January 2017, 223 patients were scheduled for cranioplasty. Re- 


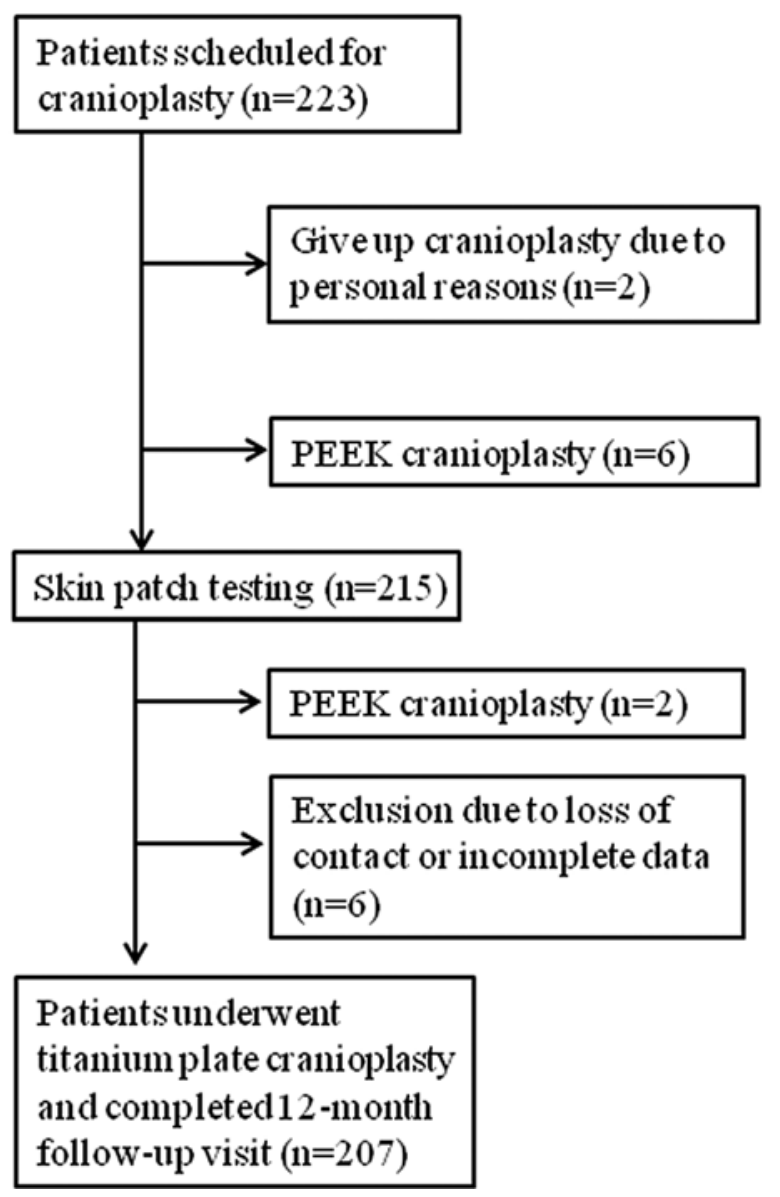

FIG. 1. Flowchart showing patient inclusion.

cords for 6 patients were incomplete due to loss of contact. Two patients refused cranioplasty for personal reasons. Six patients chose PEEK cranioplasty before patch testing. Two patients decided to receive PEEK cranioplasty after patch testing, but neither showed metal hypersensitivity in the tests. A total of 207 patients underwent titanium cranioplasty in this prospective study. Twenty-one patients received ventriculoperitoneal shunts due to the presence of hydrocephalus either at the same time of or within 12 months of cranioplasty. The flow chart for patient inclusion and demographic data are summarized in Fig. 1 and Table 1. No patient died during the study period.

\section{Patch Testing}

Skin patch tests were conducted before cranioplasty. Test results from the 207 patients are summarized in Table $2 ; 125$ patients $(60.39 \%)$ showed no hypersensitivity to any of the tested metals. Only one patient showed an uncertain allergy to titanium $( \pm)$ in this study. The most frequently identified metal hypersensitivities (skin reactions showing "+", "++", or “+++") were: Co (19.32\%), Cd (15.94\%), Zn (14.49\%), and $\mathrm{Cr}(10.63 \%)$. Fewer than $3 \%$ of patients showed allergies to $\mathrm{Sn}, \mathrm{Mo}$, or $\mathrm{Al}$, of which the frequencies were $2.42 \%, 2.42 \%$, and $0.97 \%$, respectively. Hypersensitivities to multiple metals were identified in a significant proportion of patients (Table 3). There were 52
TABLE 1. Patient demographics

\begin{tabular}{lc}
\hline \multicolumn{1}{c}{ Demographic Characteristics } & Value $(\%)$ \\
\hline No. of patients & 207 \\
\hline Male/female ratio & $155: 52$ \\
\hline Mean age, yrs & $44.60 \pm 11.60$ \\
\hline Cause of skull defect & $160(77.29)$ \\
\hline Trauma \& decompression & $41(19.81)$ \\
\hline Intracranial hemorrhage \& decompression & $4(1.94)$ \\
\hline Ischemic stroke & $2(0.97)$ \\
\hline Osteofibrous dysplasia & \\
\hline Type of skull deficiency & $165(79.71)$ \\
\hline Unilateral & $28(13.53)$ \\
\hline Bilateral & $14(6.76)$ \\
\hline Bifrontal & \\
\hline Other metal implants & $25(12.08)$ \\
\hline Orthopedic implants & $14(6.76)$ \\
\hline Intravascular devices & $36(17.39)$ \\
\hline Dental & $11(5.31)$ \\
\hline Cranioplasty implant failure & $11(5.31)$ \\
\hline Implant exposure & $4(1.93)$ \\
\hline Superficial infection & $3(1.45)$ \\
\hline Deep infection* & $0(0)$ \\
\hline Mortality &
\end{tabular}

(25.12\%) patients showing hypersensitivity to more than 1 kind of metal. Among patients with multiple metal hypersensitivities, allergies to $\mathrm{Cd}+\mathrm{Zn}(10.15 \%)$ and $\mathrm{Cd}+\mathrm{Cr}$ $(7.25 \%)$ were most commonly identified, followed by hypersensitivities to $\mathrm{Co}+\mathrm{Cd}(7.25 \%), \mathrm{Co}+\mathrm{Zn}(6.28 \%)$, and $\mathrm{Cd}+\mathrm{Cr}+\mathrm{Zn}(5.80 \%$; Table 4).

\section{Cranioplasty and Implant Failure}

Cranioplasty was performed in 215 patients. Eight patients chose PEEK reconstruction, and 207 patients received computer-designed titanium plate cranioplasty. Two patients with osteofibrous dysplasia underwent cranioplasty during the craniectomy. The remaining 205 patients underwent cranioplasty between 8 weeks and 9 months after the initial craniectomy (mean $21.54 \pm 3.17$ weeks). Significant serious adverse events, such as death, CSF leakage, intracranial hemorrhage, intracranial infection, skin flap necrosis, or refractory epilepsy, were not detected in this study. Subcutaneous hydrops was detected in $11(5.31 \%)$ patients after cranioplasty, and all cases were cured after conservative treatment. There was no impaired wound healing or prolonged fever during the perioperative periods of cranioplasty. Stitches were removed within 2 weeks after cranioplasty, and the patients were discharged for further follow-up visits. There were 27 (13.04\%) patients who required prolonged antiseizure treatment due to the presence of epilepsy after either craniectomy or cranioplasty.

During the 12-month follow-up, the 8 patients who re- 
TABLE 2. Results of patch testing before cranioplasty in 207 patients

\begin{tabular}{|c|c|c|c|c|c|c|}
\hline \multirow[b]{2}{*}{ Tested Metals } & \multicolumn{6}{|c|}{ Results } \\
\hline & - & \pm & + & ++ & +++ & Confirmed Allergies* \\
\hline Co & 153 (73.91) & $14(6.76)$ & $36(17.39)$ & $3(1.45)$ & $1(0.48)$ & $40(19.32)$ \\
\hline $\mathrm{Cd}$ & $162(78.26)$ & $12(5.80)$ & $32(15.46)$ & $1(0.48)$ & $0(0)$ & $33(15.94)$ \\
\hline $\mathrm{Zn}$ & $174(84.06)$ & $3(1.45)$ & $16(7.73)$ & $5(2.42)$ & $9(4.35)$ & $30(14.49)$ \\
\hline $\mathrm{Cr}$ & $178(85.99)$ & $7(3.38)$ & $22(10.63)$ & $0(0)$ & $0(0)$ & $22(10.63)$ \\
\hline $\mathrm{Hg}$ & $180(86.96)$ & $11(5.31)$ & $11(5.31)$ & $2(0.97)$ & $3(1.45)$ & $16(7.73)$ \\
\hline $\mathrm{Ag}$ & $183(88.41)$ & $10(4.83)$ & $11(5.31)$ & $2(0.97)$ & $1(0.48)$ & $14(6.76)$ \\
\hline $\mathrm{Mn}$ & $185(89.37)$ & $12(5.80)$ & $9(4.35)$ & $1(0.48)$ & $0(0)$ & $10(4.83)$ \\
\hline $\mathrm{Ni}$ & $190(91.79)$ & $7(3.38)$ & $4(1.93)$ & $4(1.93)$ & $2(0.97)$ & $10(4.83)$ \\
\hline $\mathrm{Pd}$ & $191(92.27)$ & $8(3.86)$ & $7(3.38)$ & $1(0.48)$ & $0(0)$ & $8(3.86)$ \\
\hline $\mathrm{Fe}$ & $194(93.72)$ & $3(1.45)$ & $8(3.86)$ & $1(0.48)$ & $1(0.48)$ & $10(4.83)$ \\
\hline Sn & $196(94.69)$ & $6(2.90)$ & $4(1.93)$ & $1(0.48)$ & $0(0)$ & $5(2.42)$ \\
\hline Mo & $198(95.65)$ & $4(1.93)$ & $5(2.42)$ & $0(0)$ & $0(0)$ & $5(2.42)$ \\
\hline Al & $201(97.10)$ & $4(1.93)$ & $2(0.97)$ & $0(0)$ & $0(0)$ & $2(0.97)$ \\
\hline $\mathrm{Ti}$ & 206 (99.51) & $1(0.48)$ & $0(0)$ & $0(0)$ & $0(0)$ & $0(0)$ \\
\hline Vaseline (control) & $207(100.00)$ & $0(0)$ & $0(0)$ & $0(0)$ & $0(0)$ & $0(0)$ \\
\hline
\end{tabular}

ceived PEEK cranioplasty all showed good outcomes, although they were not included in the final statistics. Eleven (5.31\%) of 207 patients who received titanium cranioplasty developed delayed scalp erosion and implant exposure (Table 1). None of the 11 patients showed titanium hypersensitivity in patch testing. The first implant failure occurred at 13 weeks after cranioplasty, while the last case was identified at 11 months of follow-up. The mean time of such complication occurred at $8.27 \pm 2.53$ months after cranioplasty (median 8.75 months). All 11 patients underwent reoperations and titanium plate removal. Nine patients underwent reoperations at the same institutions where the cranioplasty was performed, and the other 2 patients underwent reoperation at one of our joint facilities. The removed titanium plates and adjacent tissue samples were collected for bacteriological examinations. Bacterial infections were confirmed in 7 of 11 cases by culture examinations. Staphylococcus epidermidis and Staphylococcus aureus were found in 5 and 2 cases, respectively. The infections involved the skin, subcutaneous tissues, deep soft tissues, or meninges of the incision site. Brain tissue or any other kind of intracranial infection was not detected in this study.

The association between metal hypersensitivities and titanium implant failure is shown in Tables 3 and 4 . Patients who showed negative $(-)$ results to all tested metals had a significantly lower risk of titanium implant failure ( $\mathrm{p}$ $<0.001)$, while any confirmed metal hypersensitivities $(+$, ++ , or +++$)$ could be considered a risk factor $(\mathrm{p}<0.001$, Table 3). Patients having multiple metal hypersensitivities in patch tests, particularly for those who had confirmed allergies to 4 or 5 metals, had a statistically significantly higher chance of developing implant failure (Table 3). The odds ratio also indicated a potential association between

TABLE 3. Results of patch testing in association with titanium implant failure

\begin{tabular}{ccccc}
\hline No. of Confirmed Allergies* & No. of Pts (\%) & No. of Implant Failures (\%) & OR (95\% Cl) & $p$ Value \\
\hline Null & $125(60.39)$ & $1(0.48)$ & $0.058(0.0073-0.46)$ & $<0.001$ \\
\hline$\geq 1$ positive reaction & $82(39.61)$ & $10(4.83)$ & $17.22(2.16-137.31)$ & $<0.001$ \\
\hline 1 & $30(14.49)$ & $1(0.48)$ & $0.57(0.071-4.67)$ & $>0.99$ \\
\hline 2 & $21(10.14)$ & $1(0.48)$ & $0.88(0.11-7.24)$ & $>0.99$ \\
\hline 3 & $17(8.21)$ & $2(0.97)$ & $2.68(0.53-13.55)$ & 0.23 \\
\hline 4 & $7(3.86)$ & $3(1.45)$ & $18.00(3.44-94.25)$ & 0.0035 \\
\hline 5 & $4(1.93)$ & $2(0.97)$ & $43.33(3.58-523.56)$ & 0.0075 \\
\hline$\geq 6$ & $3(1.45)$ & $1(0.48)$ & $9.70(0.81-116.20)$ & 0.15 \\
\hline
\end{tabular}

Pts $=$ patients.

Boldface type indicates statistical significance.

* Reactions of,+++ , or +++ in patch testing were considered confirmed allergies. 
TABLE 4. The most frequently identified metallic hypersensitivities associated with titanium implant failure

\begin{tabular}{lcccc}
\hline Metal $^{*}$ & No. of Pts (\%) & No. of Implant Failures $(\%)$ & OR $(95 \% \mathrm{Cl})$ & $\mathrm{p} \mathrm{Value}$ \\
\hline None & $125(60.39)$ & $1(0.48)$ & $0.058(0.0073-0.46)$ & $<0.001$ \\
\hline Co & $40(19.32)$ & $5(2.42)$ & $3.83(1.11-13.27)$ & 0.039 \\
\hline $\mathrm{Cd}$ & $33(15.94)$ & $4(1.93)$ & $3.29(0.91-11.96)$ & 0.078 \\
\hline $\mathrm{Zn}$ & $30(14.49)$ & $3(1.45)$ & $2.34(0.59-9.40)$ & 0.20 \\
\hline $\mathrm{Cr}$ & $22(10.62)$ & $3(1.45)$ & $3.49(0.85-14.29)$ & 0.09 \\
\hline $\mathrm{Cd}+\mathrm{Zn}$ & $21(10.15)$ & $2(0.97)$ & $2.07(0.41-10.29)$ & 0.31 \\
\hline $\mathrm{Cd}+\mathrm{Cr}$ & $15(7.25)$ & $2(0.97)$ & $3.12(0.61-16.00)$ & 0.18 \\
\hline $\mathrm{Co}+\mathrm{Cd}$ & $14(6.76)$ & $2(0.97)$ & $3.40(0.66-17.56)$ & 0.16 \\
\hline $\mathrm{C}+\mathrm{Zn}$ & $13(6.28)$ & $1(0.48)$ & $1.53(0.18-12.99)$ & 0.54 \\
\hline $\mathrm{Cd}+\mathrm{Cr}+\mathrm{Zn}$ & $12(5.80)$ & $1(0.48)$ & $1.68(0.20-14.35)$ & 0.49 \\
\hline
\end{tabular}

Boldface type indicates statistical significance.

* Patients may not be allergic to the listed metals only (e.g., patients showing hypersensitivity to Co could also be allergic to $\mathrm{Cd}, \mathrm{Zn}$, or $\mathrm{Cr}$ ).

titanium implant failure and patients with more than 6 positive readings in patch tests, although the relationship was not statistically significant, probably due to the limited sample size (Table 3). Allergies to Co were more frequently detected in patch testing than allergies to other metals. Table 4 also indicates that patients showing hypersensitivities to Co had a higher risk of implant failure. The association between other metal allergies and titanium im- plant failure was not statistically significant. The possible association of other demographic characteristics, such as sex, age, the timing of cranioplasty, side of craniectomy, and history of seizure, was also analyzed, but none showed a significant relationship to titanium implant failure (Table 5). However, the powers of these statistical calculations are less than 0.80 , suggesting that further investigations with larger sample sizes are required.

TABLE 5. Confounding factors in association with titanium implant failure

\begin{tabular}{|c|c|c|c|c|}
\hline Variable & No. of Pts (\%) & No. of Implant Failures (\%) & OR $(95 \%$ Cl) & p Value \\
\hline \multicolumn{5}{|l|}{ Sex } \\
\hline Male & $155(74.88)$ & $9(4.35)$ & $1.54(0.32-7.37)$ & 0.73 \\
\hline Female & $52(25.12)$ & $2(0.97)$ & $1.95(0.32-11.99)$ & 0.61 \\
\hline \multicolumn{5}{|l|}{ Age, yrs } \\
\hline $16-40$ & $68(32.85)$ & $4(1.93)$ & $1.18(0.33-4.17)$ & 0.75 \\
\hline $41-60$ & $103(49.76)$ & $4(1.93)$ & $0.57(0.16-2.01)$ & 0.54 \\
\hline$\geq 61$ & $36(17.39)$ & $3(1.45)$ & $1.63(0.42-6.37)$ & 0.44 \\
\hline \multicolumn{5}{|l|}{ Indication for craniectomy } \\
\hline Trauma & $160(77.29)$ & 10 & $3.07(0.38-24.60)$ & 0.46 \\
\hline Intracranial hemorrhage & $41(19.81)$ & 1 & $0.39(0.048-3.14)$ & 0.70 \\
\hline Ischemic stroke & $4(1.94)$ & $0(0)$ & $1.86(0.094-36.68)$ & 1.00 \\
\hline Osteofibrous dysplasia & $2(0.97)$ & $0(0)$ & $3.38(0.15-74.64)$ & 1.00 \\
\hline \multicolumn{5}{|l|}{ Laterality of skull defect } \\
\hline Unilateral & $165(79.71)$ & $8(3.86)$ & $0.66(01.7-2.61)$ & 0.70 \\
\hline Bilateral & $28(13.53)$ & $2(0.97)$ & $1.45(0.30-7.10)$ & 0.64 \\
\hline Bifrontal & $14(6.76)$ & $1(0.48)$ & $1.41(0.17-11.86)$ & 0.55 \\
\hline \multicolumn{5}{|c|}{ Time btwn cranioplasty \& cranioplasty, mos } \\
\hline $2-3$ & $67(32.37)$ & $2(0.97)$ & $0.45(0.094-2.13)$ & 0.51 \\
\hline $4-6$ & $97(46.86)$ & $7(3.38)$ & $2.06(0.58-7.27)$ & 0.35 \\
\hline $7-9$ & $43(20.77)$ & $2(0.97)$ & $0.84(0.17-4.04)$ & 1.00 \\
\hline Metal implants other than cranioplasty & $77(37.20)$ & $3(1.45)$ & $0.62(0.16-2.40)$ & 0.74 \\
\hline Diabetes & $19(9.18)$ & $0(0)$ & $0.40(0.22-6.98)$ & 0.60 \\
\hline VP shunt & $11(5.31)$ & $1(0.48)$ & $1.86(0.21-15.99)$ & 0.46 \\
\hline Seizure & $27(13.04)$ & $0(0)$ & $0.27(0.015-4.68)$ & 0.37 \\
\hline Subcutaneous hydrops & $11(5.31)$ & $0(0)$ & $0.70(0.039-12.66)$ & 1.00 \\
\hline
\end{tabular}




\section{Discussion}

Computer-modeled titanium prostheses are important materials for cranioplasty, although implant failure, particularly exposure of the plates, remains one of the major complications. Metal hypersensitivities and their associations with implant failure have been noted in orthopedic, endovascular, obstetric, and dental devices. Previous studies have indicated that dermal hypersensitivity to metal is common and can affect up to $15 \%$ of the population. The hypersensitivity reactions are primarily $\mathrm{T}$ cell-mediated type IV delayed-type reactions that have been established in a multitude of cohort studies. The link between eczematous dermatitis and nickel was first reported by Foussereau and Laugier in $1966 .{ }^{6}$ Later studies conducted in the 1970s and 1980s indicated that the prevalence of hypersensitivity to nickel, cobalt, or chromium in patients with hip implant failure was approximately 4-fold higher than that in the general population. ${ }^{8}$ Systematic reviews also showed that the risk of developing a metal allergy was higher in patients with failed implants (OR 2.76, 95\% CI 1.14-6.70). ${ }^{7}$ Although the existing literature generally recognizes the value, the necessity of preimplantation diagnosis of metal hypersensitivity remains controversial due to a lack of evidence-based support. The metal implants for cranioplasty are usually made of titanium alloys, which may comprise titanium, aluminum, nickel, zinc, and other metals based on different manufacturing technologies. Therefore, implant failure is still possible for patients with no allergy to titanium, although the association has not been assessed in the field of neurosurgery. In this study, a prospective multicenter investigation was conducted that involved 207 patients underwent both titanium cranioplasty and preimplantation patch testing. Our study indicates that titanium prostheses are overall stable and safe for cranioplasty, and none of the recruited patients showed allergies to titanium. Eleven $(5.31 \%$ ) patients experienced delayed scalp erosion and implant exposure, and these patients underwent additional operations for implant removal. Further investigations indicated that positive reactions in patch testing, particularly multiple metal hypersensitivities, were associated with titanium implant failure.

There have been ethics concerns regarding whether patients with metal allergies should receive titanium cranioplasty. Although metal hypersensitivity has previously been noted in orthopedic, intravascular, gynecological, and dental devices, there has been no established guideline on whether implantation should be avoided. In the field of neurosurgery, this issue has never been investigated. For many years, titanium plates have been routinely used for cranioplasty around the world, and preoperational patch testing is not performed at most institutions. In this study, allergies to titanium were not detected. The results of patch tests and the possibility of implant failure were well explained to all included patients. Patients were free to choose titanium plates or other prostheses, such as PEEK, for cranioplasty.

Another key issue to consider is whether the implant failures are caused by infections rather than hypersensitivity. In this study, signs of infections or impaired wound healing were not detected during the perioperative periods of cranioplasty. Stitches were removed within 2 weeks after cranioplasty in all patients. The earliest implant ex- posure occurred at week 13 and the last at 11 months after cranioplasty, the timing of which did not fit the usual pattern of surgical infections. In addition, although bacterial infections were confirmed in 7 cases after implant removal, a factor to consider is that dermatitis, effusions, skin erosions, and consequent exposure of the implant due to hypersensitivities are easily accompanied by secondary bacterial infections.

In the current study, preoperative skin patch testing was applied to evaluate metal hypersensitivities. Patch testing is currently the gold standard for diagnosing allergic contact dermatitis on the skin, although its utility in diagnosing allergic reactions in a peri-implant environment is much less clear. Some authors have argued that the results of patch testing may not correctly reveal the immune response to implants, as cutaneous exposure to an allergen is not the same as the constant exposure in the closed environment of a metal implant. In the skin, Langerhans cells are the antigen-presenting cells, while in the closed in vivo environment, macrophages and dendritic cells may take the role. In some studies, lymphocyte transformation testing (LTT) or leukocyte migration inhibition testing has been applied to assess delayed hypersensitivity.11,14 The LTT measures the proliferation of lymphocytes from peripheral blood in the presence of a potential allergen after incubation for 7 days. The leukocyte migration inhibition test measures the migration activity of mixed-population leukocytes, as the presence of a potential allergen slows migration down. However, the procedures of LTT or leukocyte migration inhibition testing are more complicated than patch testing, and the results sometimes are questionably reproducible and not clearly relevant. ${ }^{20}$ Therefore, although patch testing may not be the ultimate gold standard for internal metal hypersensitivity reactions, it remains the most clinically used test.

Implant failure is an important complication of cranioplasty but with a relatively low incidence. During the period of this study, only 11 of 207 patients developed unsatisfactory outcomes. This leads to a limitation of the study that some powers of the statistical calculations were less than 0.80. Randomized controlled trials with larger sample sizes have been proposed to better evaluate how patch testing could sensitize individuals to react to a metal implant. However, based on the knowledge that patients with metal allergies are at higher risk of implant failure, ethics issues may arise when conducting further randomized controlled trials. Instead, comparative effectiveness research may be an option.

\section{Conclusions}

Based on our findings, we suggest routine patch testing for all patients who will undergo titanium cranioplasty. Attention shall be paid to those with allergies to multiple metals. For these patients, PEEK prostheses are an option for cranioplasty, although these implants are much more expansive than titanium plates.

\section{Acknowledgments}

This work was supported by the National Natural Science Foundation of China (NSFC grants 81471241, 81301339, 81671200 
and 81271374), and The Science and Technology Commission of Shanghai Municipality Project (16411955300, 18441903300).

\section{References}

1. Agner C, Dujovny M, Gaviria M: Neurocognitive assessment before and after cranioplasty. Acta Neurochir (Wien) 144:1033-1040, 2002

2. Al-Tamimi YZ, Sinha P, Trivedi M, Robson C, Al-Musawi TA, Hossain N, et al: Comparison of acrylic and titanium cranioplasty. Br J Neurosurg 26:510-513, 2012

3. Corliss B, Gooldy T, Vaziri S, Kubilis P, Murad G, Fargen K: Complications after in vivo and ex vivo autologous bone flap storage for cranioplasty: a comparative analysis of the literature. World Neurosurg 96:510-515, 2016

4. Crawford GH: The role of patch testing in the evaluation of orthopedic implant-related adverse effects: current evidence does not support broad use. Dermatitis 24:99-103, 2013

5. Di Stefano C, Sturiale C, Trentini P, Bonora R, Rossi D, Cervigni G, et al: Unexpected neuropsychological improvement after cranioplasty: a case series study. Br J Neurosurg 26:827-831, 2012

6. Foussereau J, Laugier P: Allergic eczemas from metallic foreign bodies. Trans St Johns Hosp Dermatol Soc 52:220 225,1966

7. Granchi D, Cenni E, Giunti A, Baldini N: Metal hypersensitivity testing in patients undergoing joint replacement: a systematic review. J Bone Joint Surg Br 94:1126-1134, 2012

8. Hallab N, Merritt K, Jacobs JJ: Metal sensitivity in patients with orthopaedic implants. J Bone Joint Surg Am 83A:428-436, 2001

9. Iwama T, Yamada J, Imai S, Shinoda J, Funakoshi T, Sakai $\mathrm{N}$ : The use of frozen autogenous bone flaps in delayed cranioplasty revisited. Neurosurgery 52:591-596, 2003

10. Jelcic N, De Pellegrin S, Cecchin D, Della Puppa A, Cagnin A: Cognitive improvement after cranioplasty: a possible volume transmission-related effect. Acta Neurochir (Wien) 155:1597-1599, 2013

11. Luque I, Leyva L, José Torres M, Rosal M, Mayorga C, Segura JM, et al: In vitro T-cell responses to beta-lactam drugs in immediate and nonimmediate allergic reactions. Allergy 56:611-618, 2001

12. Morton RP, Abecassis IJ, Hanson JF, Barber J, Nerva JD, Emerson SN, et al: Predictors of infection after 754 cranioplasty operations and the value of intraoperative cultures for cryopreserved bone flaps. J Neurosurg 125:766-770, 2016

13. Ng ZY, Ang WJ, Nawaz I: Computer-designed polyetheretherketone implants versus titanium mesh ( \pm acrylic cement) in alloplastic cranioplasty: a retrospective single-surgeon, single-center study. J Craniofac Surg 25:e185-e189, 2014

14. Nyfeler B, Pichler WJ: The lymphocyte transformation test for the diagnosis of drug allergy: sensitivity and specificity. Clin Exp Allergy 27:175-181, 1997

15. Punchak M, Chung LK, Lagman C, Bui TT, Lazareff J, Rez- zadeh K, et al: Outcomes following polyetheretherketone (PEEK) cranioplasty: systematic review and meta-analysis. J Clin Neurosci 41:30-35, 2017

16. Reddy S, Khalifian S, Flores JM, Bellamy J, Manson PN, Rodriguez ED, et al: Clinical outcomes in cranioplasty: risk factors and choice of reconstructive material. Plast Reconstr Surg 133:864-873, 2014

17. Schalock PC, Thyssen JP: Patch testers' opinions regarding diagnostic criteria for metal hypersensitivity reactions to metallic implants. Dermatitis 24:183-185, 2013

18. Teo ZWW, Schalock PC: Hypersensitivity reactions to implanted metal devices: facts and fictions. J Investig Allergol Clin Immunol 26:279-294, 2016

19. Thien A, King NK, Ang BT, Wang E, Ng I: Comparison of polyetheretherketone and titanium cranioplasty after decompressive craniectomy. World Neurosurg 83:176-180, 2015

20. Thyssen JP, Menné T, Schalock PC, Taylor JS, Maibach HI: Pragmatic approach to the clinical work-up of patients with putative allergic disease to metallic orthopaedic implants before and after surgery. Br J Dermatol 164:473-478, 2011

21. Wiggins A, Austerberry R, Morrison D, Ho KM, Honeybul $\mathrm{S}$ : Cranioplasty with custom-made titanium plates -14 years experience. Neurosurgery 72:248-256, 2013

22. Williams LR, Fan KF, Bentley RP: Custom-made titanium cranioplasty: early and late complications of 151 cranioplasties and review of the literature. Int J Oral Maxillofac Surg 44:599-608, 2015

23. Wu X, Hu J, Zhuo L, Fu C, Hui G, Wang Y, et al: Epidemiology of traumatic brain injury in eastern China, 2004: a prospective large case study. J Trauma 64:1313-1319, 2008

\section{Disclosures}

The authors report no conflict of interest concerning the materials or methods used in this study or the findings specified in this paper.

\section{Author Contributions}

Conception and design: J Hu, Sun. Acquisition of data: J Hu, Sun, Y Hu, Yuan. Analysis and interpretation of data: Sun, Y Hu, Yuan, J Yu. Drafting the article: Sun, Yuan, J Yu. Critically revising the article: J Hu. Reviewed submitted version of manuscript: Sun, Y Hu, Yuan, J Yu, Xing Wu. Statistical analysis: Sun, Y $\mathrm{Hu}$, Yuan, J Yu. Administrative/technical/material support: Sun, Y Hu, Xing Wu, Du, Xuehai Wu. Study supervision: Sun, Y Hu, Xing $\mathrm{Wu}, \mathrm{Du}$, Xuehai Wu.

\section{Correspondence}

Jin Hu: Huashan Hospital, Fudan University, Shanghai, China. hujindn@126.com. 